\title{
THE RADICAL OF A GROUP WITH OPERATORS
}

\section{GARRETT BIRKHOFF}

In a recent note (The radical of a non-associative algebra, Bull. Amer. Math. Soc. vol. 48 (1942) pp. 893-897) A. A. Albert has defined the radical of a general linear algebra, and deduced some of its properties from his theory of isotopy. The purpose of the present note is to extend this concept to groups with operators, and to derive similar properties from lattice theory.

Let $G$ be a group with a class $\Omega$ of endomorphisms including all inner automorphisms. ${ }^{1}$ By an ideal, we mean a subgroup $S$ of $G$ such that $s \in S$ and $w \in \Omega$ imply sw $\in S$. Clearly $G$ and the group identity 0 are ideals; any other ideal is called a proper ideal. We recall that the ideals of $G$ form a modular lattice ${ }^{2}$ we shall assume below that this has finite length.

We define $G$ to be prime if and only if it has no proper ideals; it is well known (and easy to prove) that $G / S$ is prime if and only if $S$ is maximal. Now let 3 be any class of groups with operators (specializing to zero algebras) which is invariant under isomorphisms, ${ }^{3}$ and let us define a simple group (with operators) to be any prime group not in the exceptional class 3 . We define a direct sum of prime groups to be semiprime, and (following Albert and others) a direct sum of simple groups to be semisimple.

We further define the $\phi$-ideal $\Phi$ of $G$ as the intersection of all maximal ideals (by analogy with the $\phi$-subgroup of a group), the radical $R$ of $G$ as the intersection of all maximal ideals $S$ such that $G / S$ is simple, and denote by $Z$ the intersection of all maximal ideals $T$ such that $(G / T) \in 3$.

THEOREM 1. The quotient group $G / S$ is semiprime if and only if $S$ contains $\Phi$.

Proof. If $G / S$ is semiprime, then it can be written as a direct sum $G / S=\left(S_{1} / S\right)+\cdots+\left(S_{r} / S\right)$, where the $\left(S_{k} / S\right)$ are prime. The $B_{i}=S_{1} \cup \ldots \cup S_{i-1} \cup S_{i+1} \cup \ldots \cup S_{r}$ are maximal ideals; and

Received by the editors February 24, 1943.

${ }^{1}$ If $G$ is commutative, $\Omega$ may be void. The case of a linear algebra is included by requiring $\Omega$ to consist of all scalar multiplications $x \rightarrow \lambda x$, all left multiplications $x \rightarrow a x$, and all right multiplications $x \rightarrow x a$. It follows that ideals and direct sums correspond.

2 In the sense of the author's Lattice theory, Amer. Math. Soc. Colloquium Publications, vol. 25, New York, 1940. This will be referred to below as [LT].

${ }^{3}$ It is to be observed that a zero algebra is not a "zero" group with operators: $\lambda x \neq 0$ for scalar operators $\lambda$. 


$$
S=\bigwedge_{i=1}^{r} B_{i} \geqq \Phi .
$$

To prove the converse, note that $\Phi$ is the meet of dual points (in the sense of lattice theory), and hence (by [LT, Theorem 4.1]) the modular lattice of ideals $X$ satisfying $\Phi \leqq X \leqq G$ is complemented. It follows (ibid, $\$ 65$ ) that we can find ideals $D_{1}, \cdots, D_{r}$ prime over $X$ such that

$$
\left(D_{1} \cup \ldots \cup D_{k-1}\right) \cap D_{k}=X \text { for all } k \text {, }
$$

whence $G / X=\left(D_{1} / X\right)+\cdots+\left(D_{r} / X\right)$, in the sense of being a direct sum.

THEOREM 2. The quotient group $G / S$ is semisimple if and only if $S$ contains $R$

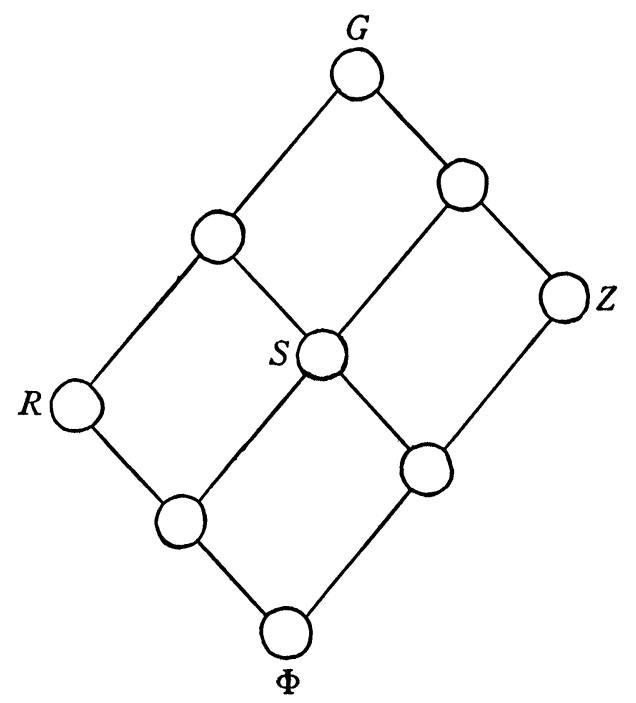

Proof. We consider the complemented modular lattice just described. "Projective" quotients (in the sense of lattice theory) are operator-isomorphic [LT, Theorem 3.16]; hence no $S$ for which $G / S$ is simple is projective with an $S$ for which $G / S$ is in 3 . It follows [LT, Theorem 4.6 and Corollary] that $R / \Phi$ and $Z / \Phi$ are "neutral" complements in $G / \Phi$. Consequently any $S$ such that $G / S$ is semiprime (let alone semisimple) fits into the lattice just described according to the scheme indicated graphically above. We have, by well known theorems, the isomorphisms 


$$
\begin{aligned}
& G / S \cong(R \cup S / S)+(Z \cup S / S), \\
& S / \Phi \cong(R \cap S / \Phi)+(Z \cap S / \Phi),
\end{aligned}
$$

where $R \cup S / S \cong R / R \cap S \cong G / Z \cup S$ is a direct sum of groups in 3 and $Z \cup S / S \cong Z / Z \cap S \cong G / R \cup S$ is semisimple. We omit the details, which are identical with those of the second part of the proof of Theorem 1 as regards representation as direct sums of prime groups, and involve [LT, Theorem 4.5], for the basic isomorphisms. Theorem 2 appears as an obvious corollary of these facts and Theorem 1.

The remarkable fact about the radical of a linear associative algebra from this point of view is that it is nilpotent. Also, the radical of a complex Lie algebra $A$ is solvable, and $[A, A]$ is nilpotent. The radical of an alternative algebra is also nilpotent. The generalization of these properties to more general classes of algebras would be extremely important.

HARVARD UNIVERSITY 